\title{
Use of Proton Pump Inhibitor and Risk of Colorectal Cancer: A Meta-analysis of Observational Studies
}

\author{
Jeong Soo Ahn, Sang Min Park, Chun Sick Eom', Sarah Kim², \\ Seung-Kwon Myung,
}

Department of Family Medicine, Seoul National University Hospital, Seoul National University College of Medicine, Seoul; ${ }^{1}$ Department of Family Medicine, Hallym University Chuncheon Sacred Heart Hospital, Hallym University College of Medicine, Chuncheon; ${ }^{2}$ Department of Family Medicine, Seoul National University Bundang Hospital, Seoul National University College of Medicine, Seongnam; ${ }^{3}$ Carcinogenesis Research Branch, Research Institute, National Cancer Center; ${ }^{4}$ Family Medicine Clinic, National Cancer Center Hospital, National Cancer Center, Goyang, Korea

Background: Previous case-control studies have reported inconsistent findings regarding the association between proton pump inhibitor (PPI) use and colorectal cancer (CRC) risk. We investigated these associations using meta-analysis.

Methods: We searched MEDLINE (PubMed), EMBASE, and the Cochrane Library in April 2011. Two evaluators independently reviewed and selected articles, based on pre-determined selection criteria.

Results: Out of 737 articles meeting our initial criteria, 5 case-control studies, which involved 120,091 participants (9,514 cases and 110,577 controls), were included in the final analyses. The overall use of PPI (used vs. never or rarely used) was not significantly associated with the risk of CRC in a fixed-effects model meta-analysis of all 5 case-control studies (odds ratio $[\mathrm{OR}], 1.08 ; 95 \%$ confidence interval $[\mathrm{CI}], 0.96$ to $1.20 ; \mathrm{I}^{2}=3.5 \%$ ). Also, in sensitivity meta-analysis by cumulative duration of PPI use, there was no association between PPI use of 1 year or longer and the risk of colorectal cancer in a fixed-effects meta-analysis (OR, 1.09; 95\% CI, 0.98 to $1.22 ; \mathrm{I}^{2}=0 \%$ ).

Conclusion: Although hypergastrinemia could be an important factor in the pathogenesis of some colorectal cancers, our study suggests that this does not lead to significant clinical risk for most PPI users. Further prospective studies or randomized controlled trials related to PPI use and colorectal cancer risk are needed to investigate this association.

Keywords: Proton Pump Inhibitors; Colorectal Neoplasms; Meta-analysis

\section{INTRODUCTION}

Received: August 31, 2011, Accepted: July 17, 2012

${ }^{*}$ Corresponding Author: Seung-Kwon Myung

Tel: 82-31-920-0479, Fax: 82-31-920-2606

E-mail: msk@ncc.re.kr

Ahn JS and Park SM contributed equally to this work.

Korean Journal of Family Medicine

Copyright (C) 2012 The Korean Academy of Family Medicine

(ㄷ) This is an open-access article distributed under the terms of the Creative Commons Attribution Non-Commercial License (http://creativecommons.org/licenses/by-nc/3.0) which permits unrestricted noncommercial use, distribution, and reproduction in any medium, provided the original work is properly cited.
Proton pump inhibitors (PPIs) are potent medications to inhibit gastric acid production, ${ }^{1)}$ and are widely used by physicians for effective management of gastrointestinal disorders including peptic ulcer disease and gastroesophageal reflux disease (GERD) ${ }^{2,3)}$ Although long-term use of these drugs is considered safe, concern has risen regarding the possible link between PPIinduced hypergastrinemia and gastrointestinal cancers, including colorectal cancer (CRC). ${ }^{4-7)}$ Serum gastrin may promote the growth of normal and malignant colonic epithelial cells, ${ }^{6)}$ and 
animal studies showed that hypergastrinemia may promote adenoma progression, which is the precursor of colorectal cancer. $^{8,9)}$

Recently, several large, nested, case-control studies were performed to clarify the relationship between PPI use and colorectal cancer risk. Most previous studies have shown that use of PPI in clinical practice was not associated with increased risk of colorectal cancer. ${ }^{10-13)}$ However, one study in the United Kingdom showed a significant association between $<2$ years of PPI therapy and the risk of colorectal cancer in univariate analysis. ${ }^{12)}$ One study in Denmark also showed a marginal increased risk of colorectal cancer among those who had taken PPIs recently. ${ }^{13)}$ As the total number of PPI prescriptions are continuously increasing world-wide and many patients require long-term PPI therapy, ${ }^{1,2)}$ potential adverse effects of PPI, including its effect on colorectal cancer risk, should be evaluated. Therefore, in the current study, we estimated the magnitude of the associations between PPI use and increased risk of colorectal cancer via meta-analysis of epidemiological studies.

\section{METHODS}

\section{Literature Search}

We searched MEDLINE (PubMed, inception to April 2011), EMBASE (inception to April 2011), and the Cochrane Central Register of Controlled Trials in the Cochrane Library (inception to April 2011) using common keywords related to proton pump inhibitors and tumor or cancer of the colorectum. The keywords used were as follows: 'proton pump inhibitors,' 'omeprazole,' 'nexium,' 'lansoprazole,' 'rabeprazole,' 'pantoprazole,' or 'esomeprazole' for the exposure factors, and 'colon cancer,' 'colon neoplasia,' 'colon neoplasm,' 'colon tumor,' 'rectal cancer,' 'rectal neoplasia,' 'rectal neoplasm,' 'rectal tumor,' 'colorectal cancer,' 'colorectal neoplasia,' 'colorectal neoplasm,' or 'colorectal tumor' for the outcome factors. We also scanned the bibliographies of relevant articles in order to identify additional studies. Only articles written in English were included in the current study.

\section{Selection Criteria}

We included observational studies and clinical trials that met all of the following criteria: 1) case-control studies, cohort studies, and randomized controlled trials. Although we planned to include epidemiological studies such as prospective cohort studies or randomized controlled trials, to date, no randomized controlled trials or cohort studies have been published. We, therefore, decided to include only case-control studies in the current study. We also included studies which 2) investigated the associations between the use of proton pump inhibitors and colorectal cancer, and 3 ) reported outcome measures with odds ratio (OR) and $95 \%$ confidence interval (CI), or values in cells of a $2 \times 2$ table (from which ORs could be calculated).

\section{Selection of Relevant Studies}

All studies retrieved from databases and bibliographies were independently evaluated by two authors of this paper (Ahn JS and Park SM). Disagreements between evaluators were resolved by discussion or in consultation with a third author (Eom CS).

\section{Assessment of Methodological Quality}

We assessed the methodological quality of included studies based on the Newcastle-Ottawa Scale (NOS) for the quality of case-control studies in meta-analyses. ${ }^{14)}$ The NOS for casecontrol studies consists of 8 items with three subscales: selection of cases and controls (4 items), comparability of cases and controls ( 1 item), and exposure ( 3 items). A "star" system of the NOS (range, 0 to 9 stars) has been developed for the assessment: each study can be awarded a maximum of one star for each numbered item within the selection and exposure categories, while a maximum of two stars can be given for the comparability category. In the current study, we considered a study awarded 7 or more stars as a high quality study, as standard criteria have not been established. The mean value for the 5 studies assessed was 8.4 stars.

\section{Main and Sensitivity Analyses}

We investigated the association between the use of proton pump inhibitors (used vs. never or rarely used, if possible) and the risk of colorectal cancer by using adjusted data as the main analysis. We also performed sensitivity analyses by methodological quality (high or low quality), cumulative duration of PPI use ( $\geq 1$ year and $\geq 5$ years), and adjusted for other CRC risk factors including nonsteroidal antiinflammatory drug (NSAID)s/aspirin and body mass index. 


\section{Statistical Analyses}

To compute a pooled OR with $95 \% \mathrm{CI}$, we utilized the adjusted OR and 95\% CIs reported in each article whenever possible. Heterogeneity in results across studies was assessed by using Higgins $\mathrm{I}^{2}$, which measures the percentage of total variation across studies. ${ }^{15)}$ Negative values of $\mathrm{I}^{2}$ were set at zero so that $\mathrm{I}^{2}$ exists between $0 \%$ (no observed heterogeneity) and $100 \%$ (maximal heterogeneity). An $\mathrm{I}^{2}$ value greater than $50 \%$ was considered as indicative of substantial heterogeneity.

We estimated a pooled OR with $95 \%$ CI based on both fixed-effects and random-effects models. When substantial heterogeneity was not observed (i.e., if $\mathrm{I}^{2} \leq 50 \%$ ), the pooled estimate calculated based on the fixed-effects model using the Woolf's (inverse variance) method was reported. When substantial heterogeneity was observed (i.e., if $\mathrm{I}^{2}>50 \%$ ), the pooled estimate was calculated based on the random-effects model using the DerSimonian and Laird. ${ }^{16)}$ We were unable to assess publication bias of the studies included in the final analysis using Begg's funnel plot asymmetry and Egger's test, because when there are few studies, the power of the tests is too low to distinguish chance from real asymmetry. We used Stata SE ver. 10.1 (Stata Co., College Station, TX, USA) for statistical analysis.

\section{RESULTS}

\section{Identification of Relevant Studies}

Figure 1 shows a flow diagram of how we identified relevant studies. A total of 737 articles were identified by searching 3 databases and through hand-searching relevant bibliographies. We excluded 70 duplicate articles and an additional 652 articles that did not satisfy the selection criteria. We reviewed the full texts of the remaining 15 articles. Among these, 10 articles were excluded in the final analysis. The main reasons for exclusion of studies during the final review were as follows $(\mathrm{n}=10)$ : data on mortality only $(\mathrm{n}=1),{ }^{17)}$ data on colorectal polyp only $(\mathrm{n}=3),{ }^{18-20)}$ or review article $(n=6) .^{7,21-25)}$ The remaining 5 case-control studies were included in the final analysis. ${ }^{10-13,26)}$

\section{Characteristics of Studies Included in the} Final Analysis

In the 5 case-control studies, we identified a total of 120,091 participants, 9,514 cases, and 110,577 controls. Table 1 shows the general characteristics of the 5 case-control studies included in the final analysis. Four studies were population-based case-control studies. $^{10-13)}$ The selected studies were published between 2007 and 2011, spanning 5 years. The range of enrollment periods (study periods) for participants was 1987 to 2005 . Two studies evaluated the association between the long-term PPI use of 5 years or longer and the risk of CRC. ${ }^{12,13)}$

\section{Overall Use of PPI and Risk of CRC}

As shown in Figure 2, the overall use of PPI (used vs. never or rarely used) was not significantly associated with the risk of CRC

\begin{tabular}{|c|c|}
\hline \multicolumn{2}{|c|}{$\begin{array}{l}\text { Identified studies from the databases using keywords and bibligraphies of relevant } \\
\text { articles }(n=737) \text { : PubMed }(n=154) \text {, EMBASE }(n=579) \text {, Cochrane }(n=4)\end{array}$} \\
\hline & Exclude duplicate articles $(n=70)$ \\
\hline \multicolumn{2}{|c|}{ Articles remaining after excluding duplicates $(n=667)$} \\
\hline & Exclude according to selection criteria $(n=652)$ \\
\hline \multicolumn{2}{|c|}{ Remaining articles $(n=15)$, full text review } \\
\hline & Excluded articles $(n=10)$ : \\
\hline$\rightarrow$ & Review $(n=6)$ \\
\hline & Data on mortality only $(n=1)$ \\
\hline & Data on colorectal polyp only $(n=3)$ \\
\hline \multicolumn{2}{|c|}{5 Case-control studies included in the final analysis $(n=5)$} \\
\hline
\end{tabular}

Figure 1. Flow diagram for identification of relevant case-control studies. 

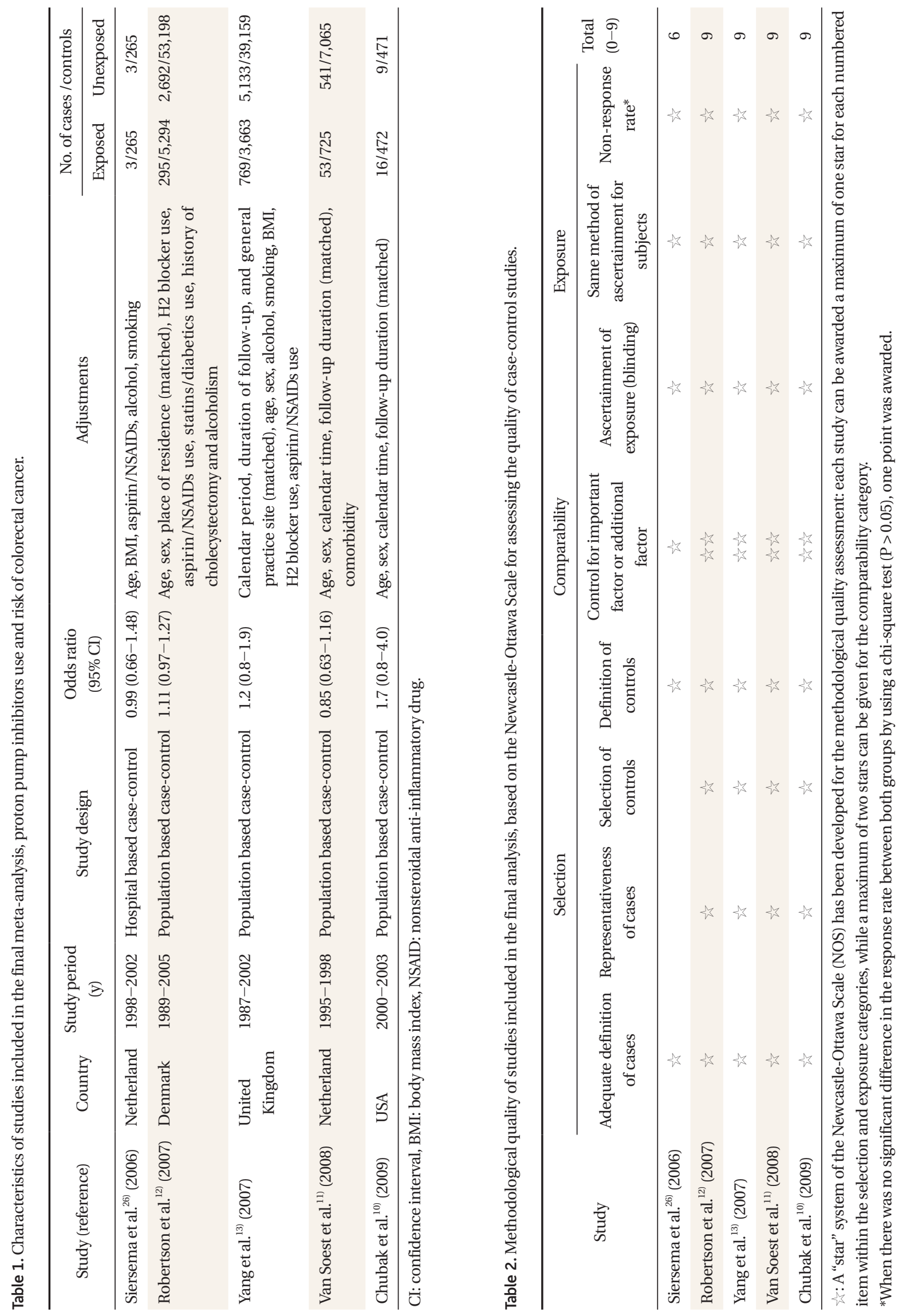


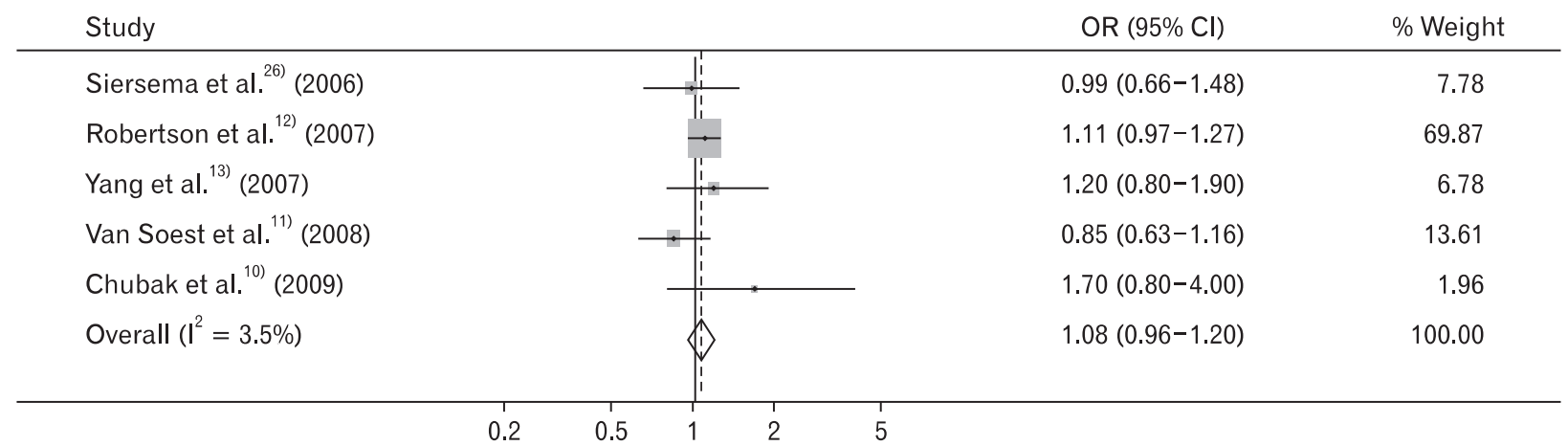

Figure 2. Use of proton pump inhibitors and risk of colorectal cancer in a meta-analysis of case-control studies $(n=5)$. The size of each square is proportional to the study's weight. Diamonds are the summary estimate from the pooled studies with $95 \%$ CI. OR: odds ratio, CI: confidence interval.

Table 3. Use of proton pump inhibitors and risk of colorectal cancer in sensitivity meta-analyses.

\begin{tabular}{|c|c|c|c|c|}
\hline Factor & No. of studies & Odds ratio $(95 \% \mathrm{CI})$ & Heterogeneity $\left(\mathrm{I}^{2}, \%\right)$ & Model used \\
\hline \multicolumn{5}{|l|}{ Methodological quality } \\
\hline High (low-bias: $\geq 7$ points)* & 4 & $1.10(0.98-1.24)$ & 0 & Fixed-effects \\
\hline \multicolumn{5}{|l|}{ Cumulative use of PPI } \\
\hline$\geq 1 \mathrm{y}$ & 3 & $1.09(0.98-1.22)$ & 0 & Fixed-effects \\
\hline$\geq 5 \mathrm{y}$ & 2 & $1.07(0.87-1.32)$ & 0 & Fixed-effects \\
\hline \multicolumn{5}{|l|}{ Adjusted for other CRC risk } \\
\hline Use of NSAIDs/aspirin & 3 & $1.11(0.98-1.25)$ & 0 & Fixed-effects \\
\hline Body mass index & 2 & $1.11(0.83-1.48)$ & 0 & Fixed-effects \\
\hline
\end{tabular}

CI: confidence interval, PPI: proton pump inhibitor, CRC: colorectal cancer, NSAID: nonsteroidal anti-inflammatory drug. *High quality study was considered a study awarded 7 or more stars, as standard criteria have not been established.

in a fixed-effects model meta-analysis of all 5 case-control studies (OR, 1.08 ; $95 \%$ CI, 0.96 to $1.20 ; \mathrm{I}^{2}=3.5 \%$ ).

Table 2 shows the methodological quality of studies included in the final analysis, based on the Newcastle-Ottawa Scale for assessing the quality of case-control studies. The range of quality scores was 6 to 9; the average score was 8.4 . The high-quality studies ( 7 or higher) included all population-based case-control studies.

\section{Sensitivity Meta-analyses}

Table 3 shows the associations between PPI use and CRC risk in sensitivity meta-analyses by methodological quality of study, cumulative duration of PPI use ( $\geq 1$ year and $\geq 5$ years), and adjustment of other CRC risk factors including NSAIDs/ aspirin or body mass index.
Among the five studies included in the final meta-analysis, only one hospital-based case-control study had a lower score of methodological quality. In sensitivity meta-analyses in high quality studies, there was no association between PPI use and CRC risk (OR, 1.10; 95\% CI, 0.98 to $1.24 ; \mathrm{I}^{2}=0 \%$ ).

In sensitivity meta-analysis by cumulative duration of PPI use, there was no association between PPI use of 1 year or longer and the risk of CRC in a fixed-effects meta-analysis (OR, 1.09; $95 \% \mathrm{CI}, 0.98$ to $\left.1.22 ; \mathrm{I}^{2}=0 \%\right)$. When we confined the cumulative duration of PPI use to $\geq 5$ years, long-term uses of PPI was not significantly associated with CRC risk (OR, 1.07; 95\% CI, 0.87 to $\left.1.32 ; \mathrm{I}^{2}=0 \%\right)$.

As for the adjustment for other CRC risk factors, use of NSAIDs/aspirin was considered in three studies, ${ }^{12,13,26)}$ and body mass index was adjusted in two studies. ${ }^{13,26)}$ In both sub-group 
meta-analysis, there was no significant association between PPI use and CRC risk.

\section{DISCUSSION}

In this meta-analysis, we assessed the association between PPI use and risk of CRC. Overall, there was no association between PPI use and CRC risk. Since the first introduction of PPIs into clinical practice in the late 1980s, PPIs were approved not only for short-term management but also for chronic use in common gastrointestinal problems such as GERD and peptic disorders. ${ }^{1-3)}$ Until now, there were several raised issues about the safety of long-term PPI use in human beings. ${ }^{7,23)}$ In particular, several mechanisms suggesting a biological link between use of the proton pump inhibitor and gastrointestinal tumor risk, including colorectal cancer, are proposed. First, the proton pump inhibitor induces hypergastrinaemia, ${ }^{27,28)}$ and long-term exposure of elevated serum gastrin could promote neoplastic growth in areas such as the stomach or colon. ${ }^{47)}$ Second, chronic suppression of gastric acid secretion may lead to enteric bacterial overgrowth in intestine, which could produce carcinogens including nitrosamines and might increase the risk of colorectal cancer. $^{25)}$

Despite this biological plausibility, we could not find statistically significant associations between PPI use and CRC risk, which is generally consistent with previous studies. This finding could be associated with the extent of hypergastrinemia and the type of gastrin involved. ${ }^{12)}$ Usually, the cumulative duration of hypergastrinemia due to PPI therapy might be shorter than other chronic conditions such as pernicious anemia or Zollinger-Ellison syndrome. ${ }^{12,29)}$ In addition, the type of gastrin that PPI therapy increased was mainly fully processed amidated gastrin, which may have a relatively weak effect on the colorectal epithelium. $^{12,13)}$

In sensitivity meta-analyses in high quality studies, there was a tendency towards a slight increase of odds ratio between PPI use and $\mathrm{CRC}$ risk, although this did not reach statistical significance. Similar results were shown in a sensitivity meta-analysis study in which an adjusted variable such as NSAIDs/aspirin use was included. In addition, when we performed the sensitivity meta-analyses, long-term PPI therapy (cumulative duration $\geq 1$ year or $\geq 5$ years) at a regular dose was also not associated with a significantly increased risk of CRC. These findings are comparable to one prospective cohort study on cancer mortality in a large cohort of patients taking omeprazole. ${ }^{17)}$ It compared the death rates of 18,000 patients who had taken omeprazole with the mortality of the general population, and there was no clear association between omeprazole use and CRC mortality.

However, we could not completely exclude the possibility of increasing CRC risk related with long-term PPI use because the lower limit of $95 \%$ CI of pooled OR was close to 1 . It is possible that, if some patients under chronic conditions of acid suppression might receive high-dose PPI therapy, this could lead to a more significant degree of additive hypergastrinemia and may have a clinical impact on increase of CRC risk. ${ }^{12)}$ In addition, although there was no definite association between PPI use and $\mathrm{CRC}$ risk in the general population, there might be a different PPI effect on CRC in a high risk population such as those with precancerous lesions or familial polyposis of the colon. Therefore, more prospective studies of PPI use and CRC risk are needed, especially focusing on high risk populations.

Our meta-analysis has several limitations. First, this study does not provide a high level of evidence because we involved only case-control studies. This topic should be re-evaluated by using enough data from prospective cohort studies. Second, we could not perform sensitivity analyses by the type of PPI because most of the studies included in these analyses did not report the type of PPI used. Third, due to the selection studies and the inevitable heterogeneity among them, pooled results of metaanalysis could incorporate the biases of individual studies and embody new sources of bias. ${ }^{30)}$

Overall, we found that there was a non-significant increase of CRC risk among PPI users in a meta-analysis of case-control studies. Although hypergastrinemia could be an important factor in the pathogenesis of some CRCs, our study suggests that this does not lead to significant clinical risk for most PPI users. However, as the adenoma-carcinoma sequence usually is a slow progress, it is still uncertain whether PPI exposure for much longer than 5 years might increase the risk of CRC. In addition, there might be a different PPI effect on CRC in a high risk population such as those with underlying hypergastrinemia or precancerous colorectal lesions. Therefore, more prospective studies or randomized controlled trials related to PPI use and 
CRC risk are needed to investigate this association.

\section{CONFLICT OF INTEREST}

No potential conflict of interest relevant to this article was reported.

\section{ACKNOWLEDGMENTS}

This work was supported by National Research Foundation of Korea Grant funded by the Korean government (grant number 2010-0004429).

\section{REFERENCES}

1. Wolfe MM, Sachs G. Acid suppression: optimizing therapy for gastroduodenal ulcer healing, gastroesophageal reflux disease, and stress-related erosive syndrome. Gastroenterology 2000;118(2 Suppl 1):S9-31.

2. Van Soest EM, Siersema PD, Dieleman JP, Sturkenboom MC, Kuipers EJ. Persistence and adherence to proton pump inhibitors in daily clinical practice. Aliment Pharmacol Ther 2006;24:377-85.

3. Bashford JN, Norwood J, Chapman SR. Why are patients prescribed proton pump inhibitors? Retrospective analysis of link between morbidity and prescribing in the General Practice Research Database. BMJ 1998;317:452-6.

4. Smith JP, Wood JG, Solomon TE. Elevated gastrin levels in patients with colon cancer or adenomatous polyps. Dig Dis Sci 1989;34:171-4.

5. Wong K, Beardshall K, Waters CM, Calam J, Poston GJ. Postprandial hypergastrinaemia in patients with colorectal cancer. Gut 1991;32:1352-4.

6. Watson SA, Morris TM, McWilliams DF, Harris J, Evans $\mathrm{S}$, Smith A, et al. Potential role of endocrine gastrin in the colonic adenoma carcinoma sequence. Br J Cancer 2002;87: 567-73.

7. La Vecchia C, Tavani A. A review of epidemiological studies on cancer in relation to the use of anti-ulcer drugs. Eur J Cancer Prev 2002;11:117-23.

8. Watson SA, Smith AM. Hypergastrinemia promotes adenoma progression in the APC(Min-/+) mouse model of familial adenomatous polyposis. Cancer Res 2001;61:62531.

9. Wu H, Rao GN, Dai B, Singh P. Autocrine gastrins in colon cancer cells Up-regulate cytochrome c oxidase $\mathrm{Vb}$ and downregulate efflux of cytochrome $\mathrm{c}$ and activation of caspase-3. J Biol Chem 2000;275:32491-8.

10. Chubak J, Boudreau DM, Rulyak SJ, Mandelson MT. Colorectal cancer risk in relation to use of acid suppressive medications. Pharmacoepidemiol Drug Saf 2009; 18:540-4.

11. van Soest EM, van Rossum LG, Dieleman JP, van Oijen MG, Siersema PD, Sturkenboom MC, et al. Proton pump inhibitors and the risk of colorectal cancer. Am J Gastroenterol 2008;103:966-73.

12. Robertson DJ, Larsson H, Friis S, Pedersen L, Baron JA, Sorensen HT. Proton pump inhibitor use and risk of colorectal cancer: a population-based, case-control study. Gastroenterology 2007;133:755-60.

13. Yang YX, Hennessy S, Propert K, Hwang WT, Sedarat A, Lewis JD. Chronic proton pump inhibitor therapy and the risk of colorectal cancer. Gastroenterology 2007; 133:748-54.

14. Wells GA, Shea B, O'Connell D, Peterson J, Welch V, Losos M, et al. The Newcastle-Ottawa Scale (NOS) for assessing the quality of nonrandomised studies in meta-analyses [Internet]. Ottawa: Ottawa Hospital Research Institute [cited 2011 Apr 30]. Available from: http://www.ohri.ca/programs/clinical_ epidemiology/oxford.htm.

15. Higgins JP, Thompson SG. Quantifying heterogeneity in a meta-analysis. Stat Med 2002;21:1539-58.

16. DerSimonian R, Laird N. Meta-analysis in clinical trials. Control Clin Trials 1986;7:177-88.

17. Bateman DN, Colin-Jones D, Hartz S, Langman M, Logan RF, Mant J, et al. Mortality study of 18000 patients treated with omeprazole. Gut 2003;52:942-6.

18. Singh M, Dhindsa G, Friedland S, Triadafilopoulos G. Long-term use of proton pump inhibitors does not affect the frequency, growth, or histologic characteristics of colon adenomas. Aliment Pharmacol Ther 2007;26:1051-61. 
19. Hsu WH, Wu IC, Kuo CH, Su YC, Lu CY, Kuo FC, et al. Influence of proton pump inhibitor use in gastrointestinal polyps. Kaohsiung J Med Sci 2010;26:76-83.

20. Freeman HJ. Proton pump inhibitors and an emerging epidemic of gastric fundic gland polyposis. World J Gastroenterol 2008;14:1318-20.

21. Freston JW. Long-term acid control and proton pump inhibitors: interactions and safety issues in perspective. Am J Gastroenterol 1997;92(4 Suppl):51S-5.

22. Joshi SN, Gardner JD. Gastrin and colon cancer: a unifying hypothesis. Dig Dis 1996;14:334-44.

23. Garnett WR. Considerations for long-term use of protonpump inhibitors. Am J Health Syst Pharm 1998;55:2268-79.

24. Nealis TB, Howden CW. Is there a dark side to long-term proton pump inhibitor therapy? Am J Ther 2008;15:536-42.

25. Laine L, Ahnen D, McClain C, Solcia E, Walsh JH. Review article: potential gastrointestinal effects of long-term acid suppression with proton pump inhibitors. Aliment Pharmacol Ther 2000; 14:651-68.

26. Siersema PD, Yu S, Sahbaie P, Steyerberg EW, Simpson PW,
Kuipers EJ, et al. Colorectal neoplasia in veterans is associated with Barrett's esophagus but not with proton-pump inhibitor or aspirin/NSAID use. Gastrointest Endosc 2006;63:581-6.

27. Allen JM, Adrian TE, Webster J, Howe A, Bloom SR. Effect of single dose of omeprazole on the gastrointestinal peptide response to food. Hepatogastroenterology 1984;31:44-6.

28. Lanzon-Miller S, Pounder RE, Hamilton MR, Ball S, Chronos NA, Raymond F, et al. Twenty-four-hour intragastric acidity and plasma gastrin concentration before and during treatment with either ranitidine or omeprazole. Aliment Pharmacol Ther 1987;1:239-51.

29. Talley NJ, Chute CG, Larson DE, Epstein R, Lydick EG, Melton LJ 3rd. Risk for colorectal adenocarcinoma in pernicious anemia: a population-based cohort study. Ann Intern Med 1989;111:738-42.

30. LeLorier J, Gregoire G, Benhaddad A, Lapierre J, Derderian F. Discrepancies between meta-analyses and subsequent large randomized, controlled trials. N Engl J Med 1997;337:53642. 